\title{
A Morphological Approach to the Design of Complex
}

\section{Objects}

\author{
R. Sarabia ${ }^{1}$, A. Jimeno-Morenilla ${ }^{1}$, R. Molina-Carmona ${ }^{2}$ \\ ${ }^{1}$ Department of Computer Science Technology and Computation \\ ${ }^{2}$ Department of Computer Science and Artificial Intelligence \\ University of Alicante, Apdo. Correos 99, 03080 Alicante, Spain. \\ Phone: +34965903400 Fax: +34965903902 \\ rsp1@alu.ua.es, jimeno@dtic.ua.es,rmolina@dccia.ua.es
}

\begin{abstract}
Background. The Surface-Trajectory model gives solution to some of the problems presented by the general geometric models where the design of an object is separated from its manufacture. In fact, in this model, the internal representation of objects is made up of machining trajectories. As the display systems usually need triangles to represent the objects, a process of triangulation is needed to visualise them. In other words, a secondary surrface model is needed to display the objects.

Method of Approach. The following is a geometric model, which maintaining the philosophy of the Surface-Trajectory model, encapsulates the calculation of the machining process from the formal framework that provides the set theory and the mathematical morphology.
\end{abstract}

Results. The model addresses the process of design objects by assimilation of a machining process by giving solutions to the design of complex objects and 
arithmetic to support the generation of trajectories of manufacturing. The design model looks like the craft work of a sculptor designing their pieces by hand with their tools. It also gives a direct solution to the problems of the generation of trajectories since they are already defined at the design phase.

Conclusions. The model is generic and robust, there are no special cases or complex objects in which the model does not provide a correct solution. It also naturally incorporates the realistic display of the machined objects in a quickly and accurately way.

Keywords: mathematical morphology, machining, geometric model

\section{Introduction}

A geometric model is a set of information, data structures, operations and tools oriented to the design of geometric objects. The features of a CAD/CAM system are largely determined by the underlying geometric model, as it is responsible for representing the objects and to perform the basic manipulation.

At present, there are essentially two general types of representations in solid modeling: boundary representations (B-REP) and constructive solid geometry (CSG). Both models of representation have interesting features, but they also have some major drawbacks which essentially are:

- The design of complex objects is usually complicated. In the case of the CSG model, the limited number of primitives can make it unable to design free surfaces. In the case of the B-REP model, the design of free surfaces is usually easier but 
their modification and adjustment (usually by manipulating the surface control points) are hardly intuitive.

- In the B-REP model, the objects are made up of surface of any type. The internal representation is usually heterogeneous to represent any type of surface. For instance, a free trimmed surface can be represented as a free surface (e.g. a NURB) and some kind of curve on the parameter space. However, a sphere can be represented as a quadratic surface. Some other methods use a discretization (an approximated polygonal mesh) so that the representation is homogeneous, but there is a lose of accuracy and the geometrical operations are complex. In the case of the CSG model, volumes are used instead of surfaces. In this case, a secondary superficial model is needed to display the objects. Therefore, the problem of triangulation arises.

- With regard to the process for manufacturing objects, the method of obtaining the machining trajectories from the geometric shapes is not trivial at all. In fact, this method often becomes the bottleneck of the design and manufacturing systems. This is because the user has complete freedom in the design, which is usually inconsistent with the restrictions imposed by the manufacture through the use of numerically controlled machines.

In general, the existing models completely separate the design process from the manufacturing one. This option allows the object design to users who are unfamiliar with the manufacturing processes. As a consequence, after the design phase, a subsequent set of complex operations is needed to obtain the machining paths, including offset calculation, machining strategies and so on [1-3]. In these cases, the quality of the manufactured pieces is usually affected because the strategies for the 
trajectories generation may not be suitable for the designed objects. So, the final object does not represent the original design with enough precision.

There are some pieces for which the models B-REP or CSG specially present design difficulties. In figure 1 a complex example of representation through B-REP modeling is presented. The design with CSG is simpler. However, for both cases, the calculations of the machining paths are highly complex.

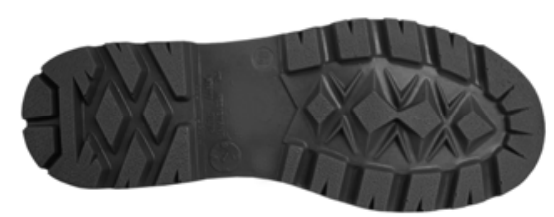

Figure 1. Example of a complex model

The mathematical morphology has its origin in the requirements of an industrial process such as the study of the characteristics of certain minerals, in order to know their properties in view of abrasion, erosion and extraction by mechanical means [4]. Nevertheless, it is not until in [5] when the morphological framework is related for the first time with the description of machining processes in two dimensions. The mathematical morphology, based on the set theory, is not subject to any spatial restrictions, always obtaining accurate and robust results [6, 7]. The SurfaceTrajectory model presented in [8] describes the objects directly through manufacturing operations, basing the definition of objects in tool trajectories. So, the pieces are designed as they are manufactured and their internal representation is made up of a set of machining trajectories traced on free surfaces. As a consequence, there 
is not a complete representation of the objects as a set of triangles. As the display systems usually need the triangles to represent the objects, a process of triangulation is needed to visualise them. This set of triangles is usually referred as a secondary surface model to display the objects, as it is only needed for this purpose. The process of triangulation is usually complex and prone to errors.

A new specialized model of representation of machined pieces using milling is presented, meaning the continuation of the Surface-Trajectory model philosophy. It integrates the process of machining calculation with the display of machined objects, all from base shapes and machining trajectories. The presented model avoids the deficiencies of the Surface-Trajectory model, providing solutions to both the problem of machining calculation and the $2 \mathrm{D} / 3 \mathrm{D}$ visualization of the machined objects in a quick and accurate way, using the formal framework provided by the mathematical morphology.

\section{Morphological model of representation}

In the Surface-Trajectory model, a piece design is given by a set of free surfaces and a series of tool trajectories [6]. The surfaces and trajectories simply and accurately define each design. The analogy between the design and the machining processes is obvious: in the machining process, the geometry of an object (piece) is also described by the geometry of another object (tool). In this model, the solution to the model representation problem needs the use of a secondary mathematical model that can describe shapes from basic geometries and provide precise solutions to the process, 
making the geometric aspects of the manufacturing process independent from the purely physical aspects derived from the use of a particular technology.

The mathematical morphology is based on the set theory, describing geometric shapes from simpler ones. Sets represent object shapes in a n-dimensional space (i.e. not limited to a specific number of dimensions) and morphological operations represent geometric relationships between the points in the sets. This theory allows formally obtaining quick and accurate results [9-12].

The proposed model takes the definitions and operations of the Mathematical Morphology paradigm as starting point, due to the fact that it presents properties that can be put on a level with the machining process [13]. Specifically, it allows the specification of pieces and tools through the use of point sets and operations describing geometric processes of cutting and reconstruction (erosion and dilation respectively). These operations are the basis for defining new morphological operations that facilitate the design of traditionally complex objects such as the one shown in figure 1.

The main advantage of the proposed model lies however, in the idea that the display calculations are already included in the machining calculations, so the integrity of the calculation-display process is guaranteed.

\subsection{Morphological modeling of machining process}

The classical morphological model has a non-deterministic nature, defined over elements of a set without order restrictions in the access to these elements. For machining purposes, the new morphologic operations will be restricted to support an 
order. The order of the morphology operation is important because it will represent the tool trajectory. For this reason, a redefinition in some aspects of the morphological model is initially needed to be suitably adapted to the characteristics of the design processes through machining. Specifically, in this section an order relation between sets elements will be incorporated so that a sequence of operations could be established and, therefore, a deterministic component is added to the morphological paradigm. Another important consideration is that only the boundary of operations will be considered, since the tool always attacks the material from outside in a mechanizing process.

Let $\mathrm{E}$ be the domain where the sets to be treated are defined. In the case of twodimensional objects $\mathrm{E} \equiv \mathrm{R}^{2}$ and for three-dimensional objects $\mathrm{E} \equiv \mathrm{R}^{3}$. In general $\mathrm{E} \equiv \mathrm{R}^{\mathrm{n}}$. Let $\mathrm{X} \subseteq \mathrm{E}$ be a subset of $\mathrm{E}$. So, in geometric terms, $\mathrm{X}$ is a two-dimensional or threedimensional object, for instance.

A function $\operatorname{Fr}(\mathrm{X})$ is defined to relate a set (i.e. an object) to its border, so that all the points belonging to the object contour are obtained (see equation 1).

$$
\begin{aligned}
& \text { Fr: } P(E) \rightarrow P(E) \\
& \operatorname{Fr}(X)=\{p \in X, p \text { is a contour point of } X\}
\end{aligned}
$$

The erosion operation in mathematical morphology can be defined according to the equation 2. Descriptively, this operation is classically defined as the place of centre positions of the structuring element $\mathrm{B}$ when it is forced to be inside an $\mathrm{X}$ set.

$$
X \square B=\left\{y \in E, B_{y} \subseteq X\right\}
$$


where $\mathrm{X}$ is the target object (the piece in the model), and $\mathrm{B}_{\mathrm{y}}$ the structuring element centered at point $y$ (the tool in the model).

For solid objects, that is, those objects that do not contain holes inside, the contour of the erosion can be defined as the centers of the structuring element when it touches the inner frontier of the object. In this context, the erosion operation in mathematical morphology can be redefined according to the equation 3 .

$$
\operatorname{Fr}(X \square B)=\left\{y \in E: B_{y} \subseteq X \wedge B_{y} \cap \operatorname{Fr}(X) \neq \varnothing\right\}
$$

\section{Instant basic operations}

A morphological operation will be divided into a sequence of unitary or basic operations. This sequence will guarantee the resulting order of the whole operation. Since every basic operation will correspond to a particular position of a tool along a trajectory that is performed during a period of time, we call them instant basic operations.

Let define the instant basic operator $\square_{\Gamma(k)}$ for the instant $k$ as follows:

$$
X \square_{\Gamma(k)} B=y \in E: y=\operatorname{dist}_{\vec{v}}(B, X \bullet \Gamma(k)) \cdot \vec{v} \wedge B_{y} \cap X \neq \varnothing
$$

where $X$ is the target object, $B$ the structuring element, $\Gamma(k)$ represents an homogeneous transformation matrix in $R^{n+1} \times R^{n+1}\left(R^{4} \times R^{4}\right.$ for a three-dimensional space) obtained for a particular $k$ real value; and dist $_{\vec{v}}$ is the Euclidean distance between the structuring element and the transformation of the $X$ object (post- 
multiplying every element of the $X$ set by the homogeneous transformation matrix $\Gamma(k))$ computed in the direction addressed by the $\vec{v}$ vector.

In other words, this operation obtains the structuring element centre when it touches the $X$ boundary following the $\vec{v}$ direction. The concept is illustrated in figure 2. In that example, an object $X$ is transformed applying a $2 \mathrm{D}$ rotation matrix over its centre $c$. For this case, $k$ could represent the number of degrees in that transformation matrix, so its values are in the $[0,2 \pi)$ range. Once the object is transformed (figure 2.c), the distance between $B$ and $X$ in the $\vec{v}$ direction is applied to the $B$ centre in order to obtain the result of the instant basic operation (that is, the $y$ point). For different and ordered real $k$ values (using the $<$ relation in $R$ ), we will obtain a new set of structuring element centres that touch the $X$ boundary. These centres will be also ordered in the geometric space due to the use of different rotation matrixes.

It is important to notice that the only geometric calculations implied in the basic operations are a homogeneous transformation and the Euclidean distance. These simple operations make the calculations very robust, due to the fact that they are not prone to numerical or geometric errors. 


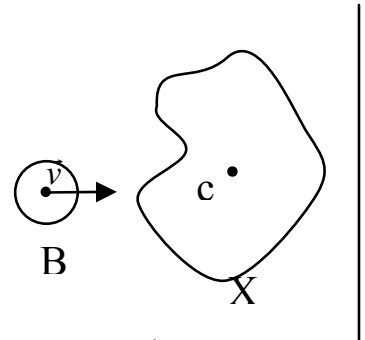

a)

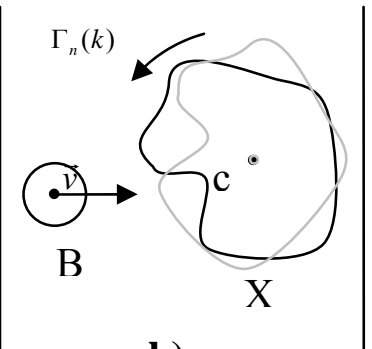

b)

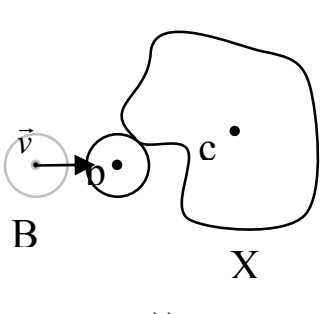

c))

Figure 2. Geometric description of an instant basic dilation. a) Initial position. b) $X$ transformation. c) Distance computing.

Starting with the instant basic operation a new operation has been created which establish the condition that the structuring element is included in the original set. If the structuring element cannot be placed into the set X (for example, when the size of $\mathrm{X}$ is less than that of $\mathrm{B}$ ), then the erosion iteration can not be applied, thus not giving any point. Erosion with trajectory is defined as the set of points obtained by repeated application of the instant erosion $\square_{\Gamma(k)}$ for the real domain $[0,1]$.

$$
X \square_{\Gamma} B=\bigcup_{k \in[0,1]}\left(X \square_{\Gamma(k)} B\right)=\left\{y \in E: B_{y} \cap \operatorname{Fr}(X) \neq \varnothing \wedge B_{y} \subseteq X\right\}
$$

The homogeneous parametric transformation $\Gamma$ should ensure an inside path through the object $\mathrm{X}$ in the normalized space $[0,1]$. If the real variable $k$ takes successive values in the interval, the boundary of the complete erosion of the object is obtained as a result, since all the centers of the structuring element when touching the object will have been obtained. It follows that the boundary of the morphological erosion and the erosion with trajectory will coincide. In general it is met: 


$$
X \square{ }_{\Gamma} B=F r(X \square B) \subseteq X \square B
$$

The erosion with trajectory allows the control of the order to obtain points in the final set. Given the relation of partial order $\leq$ in $\mathrm{R}$, an ordered succession of parametric values of $k$ in the interval $[0,1]$ will also lead to obtain an ordered collection of structuring element centers as the movement defined by the homogeneous transformation $\Gamma$ is performed. The result of applying the operation of morphological erosion on an object can be seen in figure 3. The black part correspond to the classical operation, the frontier is computed by means of the associated trajectory-based operator.

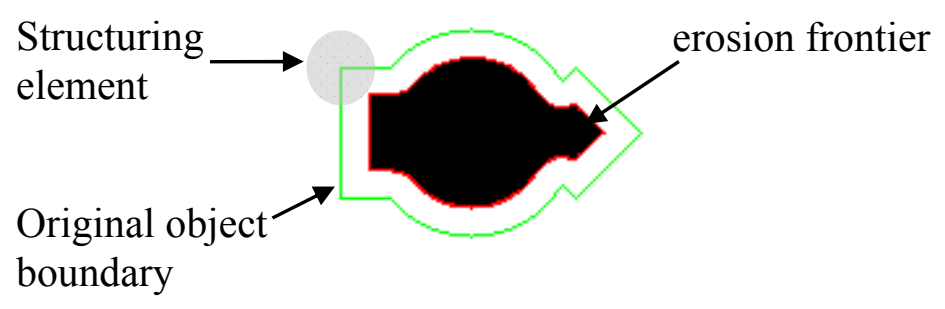

Figure 3. Example of a morphologic erosion.

The concept of morphological erosion is closely related to the process of removing material in a milling operation. The machining process using a tool can be therefore interpreted as a morphological operation of erosion, where a structural element touches an object, following in a given direction. The equivalence with the machining process is direct: in a real machining process, the structuring element would be the tool, and the object would be the material to be machined. 
However, it is important to remember that machining processes are not only dedicated to mechanize closed trajectories (equivalent to the morphological erosion) but also to mechanize open or partial paths, which do not correspond exactly with the erosion, as it stands. In this type of process, the entry and the exit of the tool also performs a work of milling on the object that is logically represented in the final result (figure 4).

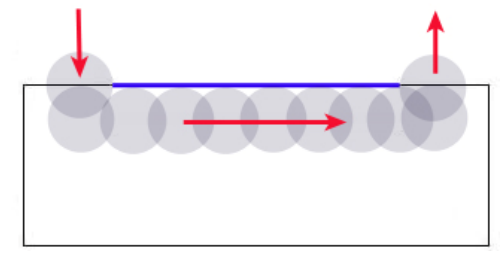

Figure 4. Example of machining a piece with a circular tool following an open trajectory

It is therefore necessary to broaden the definition of the complete morphological erosion defining a new morphological operation of partial erosion. This new operation will allow the formal definition of tool offsets for open trajectories, in which the trajectories of entry and exit of the structuring element have their formal representation in the solution.

\subsection{Regularized partial erosion}

The partial erosion partially covers the parametric space $\mathrm{k}$, constituting a subset of the complete erosion: 


$$
\mathrm{X} \square_{P \Gamma} \mathrm{B} \subseteq \mathrm{X} \square \Gamma \mathrm{B}
$$

However, the partial erosion, unlike the complete erosion, has not a direct equivalent in the machining process. In figure 5 the final result of applying the partial morphological erosion to a subset of the rectangle points can be seen.

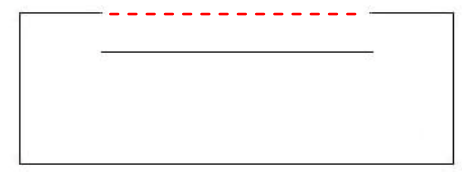

Figure 5. Partial morphological erosion as a subset of the complete erosion.

While this result is formally correct from, the solution is not valid in the applied context of design through a mechanization system. The partial erosion of intervals belonging to ordered sets of points is not possible in the context of the classical mathematical morphology, given the lack of an order relation. To give the concept of partial erosion a practical sense, it is necessary therefore to redefine it as a partial regularized operation, that is, a partial operation adapted to the implicit requirements of the process of machining a piece with a tool.

As a previous step, let us remember the concept of dilation operation in mathematical morphology, defined by the following expression::

$$
X \oplus B=\left\{y \in E, B_{y} \cap X \neq \varnothing\right\}
$$


In descriptive terms, this operation can be defined as the place of the positions of the centre of the structuring element $\mathrm{B}$ when it touches the set $\mathrm{X}$. An example of dilation of a simple line can be seen in figure 6 . For a better conceptual understanding, let us imagine that the line has a certain thickness and it dilated at all its points, so that a shape like the one in the figure is obtained.

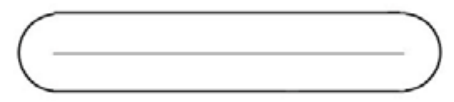

Figure 6. Dilation of simple line in two dimensions.

From the machining point of view, the regularized partial erosion of an object can now be defined as the subtraction of the dilated trajectory from the object to erode (figure 7).

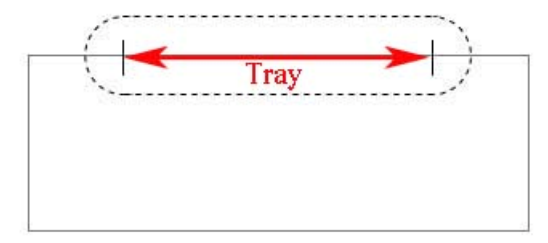

Figure 7. Dilated erosion trajectory

Figures 6 and 7 show an example of dilated trajectory. The result of applying the subtraction of the dilated trajectory from the object is shown in figure 8: 

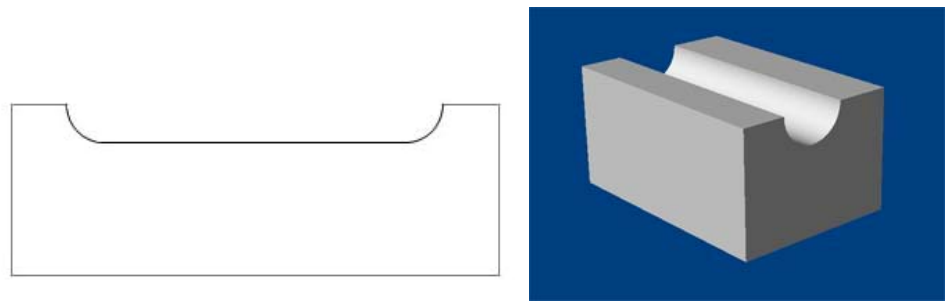

Figure 8. Two-dimensional and three-dimensional view of the result of the regularized partial erosion of a trajectory

All in all, given an object $\mathrm{X}$ and an erosion trajectory $\mathrm{T}$ defined on it, the regularized partial erosion of the object $\mathrm{X}$ by a tool $\mathrm{B}$, is the result of subtracting from the object $\mathrm{X}$ the dilation of the trajectory $\mathrm{T}$ with the radius of the tool $\mathrm{B}$. Formally, the regularized partial erosion is:

$$
\mathrm{X} \Theta_{\Gamma} \mathrm{B}=\mathrm{X} \square\left(T \oplus_{\Gamma} \mathrm{B}\right) ; T \subseteq \operatorname{Fr}(X)
$$

where $\sim$ stands for the set subtraction.

The equation 9 gives as a result the regularized partial erosion. In the next section, the calculation of the frontier of this partial erosion will be shown.

\section{Implementing the secondary model of representation}

As seen in previous sections, the model is defined by means of morphological operations on predefined shapes. For this reason, the only required information for the model is based on erosion paths and objects. The definition of the model is made on 
the continuous field so if you want to represent on screen the result of regularized partial erosions, is necessary to provide a discrete model of representation. This section provides a simple and efficient method used to show on screen the frontier of any regularized partial erosion.

Due to the fact that all the definitions in mathematical morphology are based on operations on sets of points, the implementation is done using simple structures for point storage and functions to describe the geometric transformations between point sets. The implementation of the morphological operations is, therefore, compact and precise. This section defines the morphological operations from the implementation point of view. For simplicity, the case of two dimensions is addressed initially to conclude with the model representation in three dimensions.

\section{Secondary two-dimensional model.}

The dilation of the erosion trajectory of a tool on a segment of a two-dimensional object can be described geometrically from the calculation of the parallel on both sides of the line, using the tool radius as the parallel distance, as figure 9 shows.

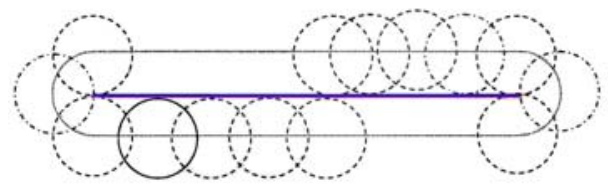

Figure 9. Dilation of erosion path from the parallel

While the dilation is done on both sides of the path, the outer part of the dilation has no influence in the erosion process because this part does not come into contact 
with the eroded element. That is why, for a practical purpose, only to the inner part of the dilation is necessary. Geometrically, the calculation of the subtraction of the obtained dilation from the object to be eroded is immediate, thanks to the fact that this solution is based on set theory. The result of this subtraction is given by the subset of points belonging to the object that does not come into contact with the structuring element, as well as the points derived from the calculation of the dilation of the trajectory

The result of the regularized erosion operation is a set of points directly obtained from the morphological method. However, as it is an infinite set of points, its representation requires a discretization, that is, a meshing process based on geometric methods. The discretization is naturally obtained thanks to the order relation that was introduced in section 2 .

The problem of representing a two-dimensional dilation is quite simple because its solution just consists in discretizing the set $\mathrm{X}$ in $\mathrm{n}_{\text {seg }}$ segments and calculating every point $\mathrm{P}$ in the parallel of each segment $\overline{p_{i} q_{i}}$. The parallel set of points is defined as follows:

$$
P=\left\{\begin{array}{l}
p^{\prime}{ }_{i_{j}}=p_{i_{j}}+R \cdot \frac{\left(p_{i} y-q_{i} y, q_{i X}-p_{i X}\right)}{\sqrt{\left(q_{i} X-p_{i} X\right)^{2}+\left(q_{i} y-p_{i} y\right)^{2}}} \\
: p_{i_{j}} \in X ; \forall j=0, \ldots, d_{i} ; \forall i=0, \ldots, n_{\text {seg }}
\end{array}\right\}
$$

where $i$ is the number of segment to erosion, $p_{i}$ and $q_{i}$ the initial and final points of segment $\mathrm{i}, \mathrm{p}_{\mathrm{ij}}$ is every discrete point for the segment $\overline{p_{i} q_{i}}, \mathrm{~d}_{\mathrm{i}}$ the number of discrete 
points on segment $\mathrm{i}$ and $\mathrm{R}$ the radius of the considered tool. At the end points, as figure 10 shows, an angular sweep of normals is done to obtain the dilation of the trajectory at these points.

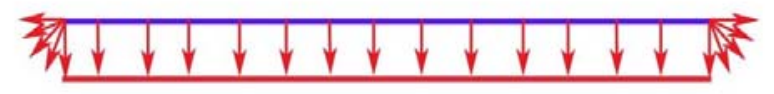

Figure 10. Angular sweep for calculating the two-dimensional dilation

Formally, the sweep $\left(\mathrm{B}_{\mathrm{j}}\right)$ for one extreme $\mathrm{p}_{\mathrm{j}}$ is defined as the point set resulting of rotating $\mathrm{n}$ times the point $\mathrm{p}$ ' an angle $\alpha_{\mathrm{i}}$, where $\mathrm{n}$ is the desired precision:

$$
\begin{array}{r}
B_{j}=\left\{p_{1}, p_{2}, . . p_{n}\right\}=\bigcup_{i=0, . . n}\left(\operatorname{Rot}\left(p_{j}^{\prime}, \alpha_{i}\right)\right) \\
\alpha_{i}=\frac{i \cdot \alpha}{n} ; i=0, . . n \quad \alpha=\operatorname{ang}\left(\text { vector }_{n}, \text { vector }_{p}\right)
\end{array}
$$

This sweep must also be done between segments forming angles greater than 0 depending on whether the parallel is on one side or on the other of the segment. Once the point set of the parallel $(\mathrm{P})$ and the point set obtained through sweeping $\left(\mathrm{C}_{\mathrm{B}}\right)$, the dilation is defined as:

$$
E_{p 2 D}=P \cup C_{B} ; \quad C_{B}=\bigcup_{j=0, . t}\left(B_{j}\right)
$$


where $t$ is the number of sweep steps. The algorithm in figure 11 geometrically represents the morphological dilation in two dimensions.

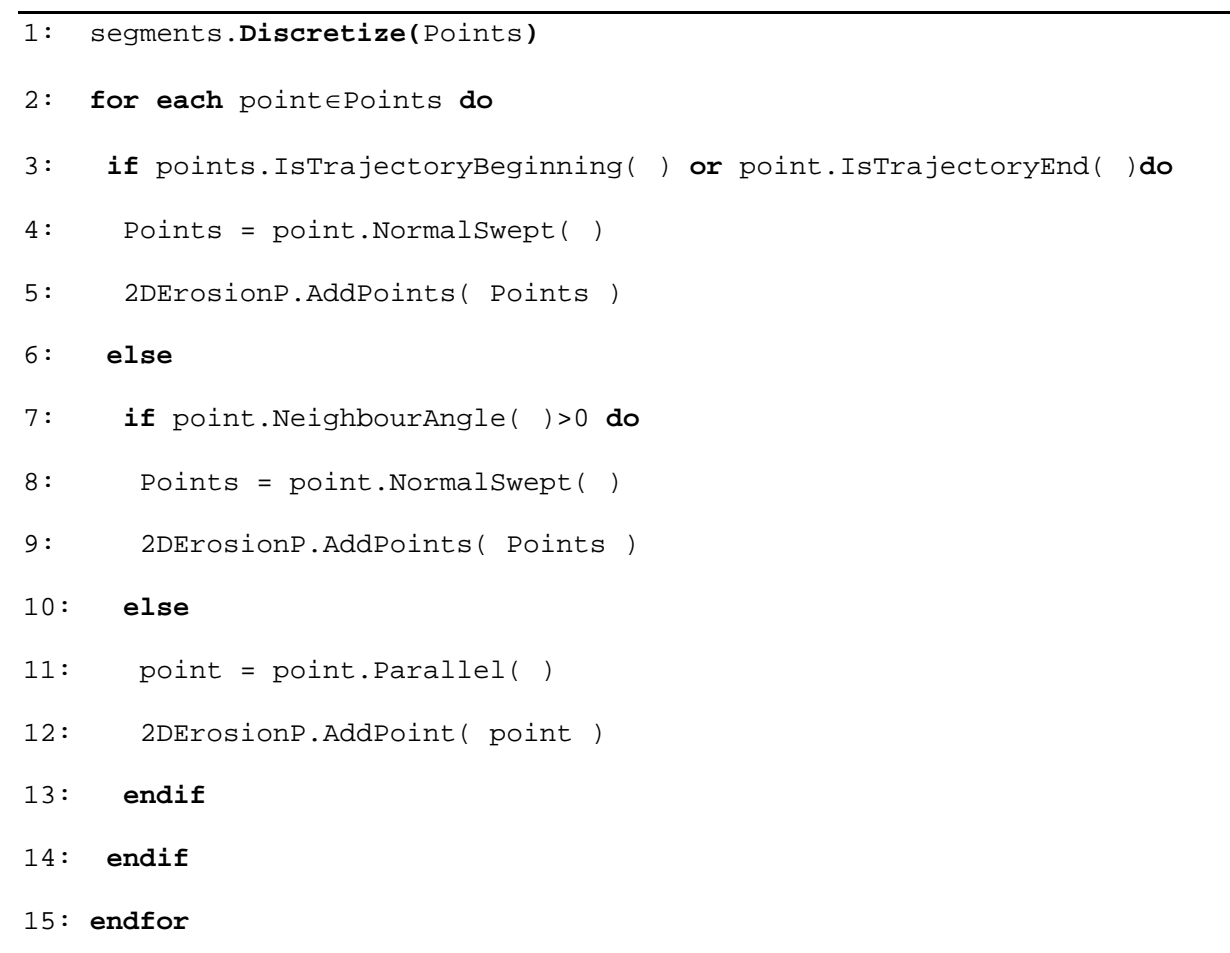

Figure 11. Dilation algorithm in two dimensions.

Finally, the partial erosion can be obtained as the subtraction of the obtained dilation from the object to be eroded. As explained before, this calculation is facilitated by the set theory. Notice that the final result of the regularized partial erosion is the frontier of the mechanized object, so it is the boundary representation that allows the direct display of the object.

In figure 12 an example of the frontier of the regularized partial erosion in two dimensions can be observed. On the left, the dashed line represents the path to be 
eroded by the tool. In the middle, the dilation of the trajectory is represented. On the right, the final result for the regularized partial erosion is shown.

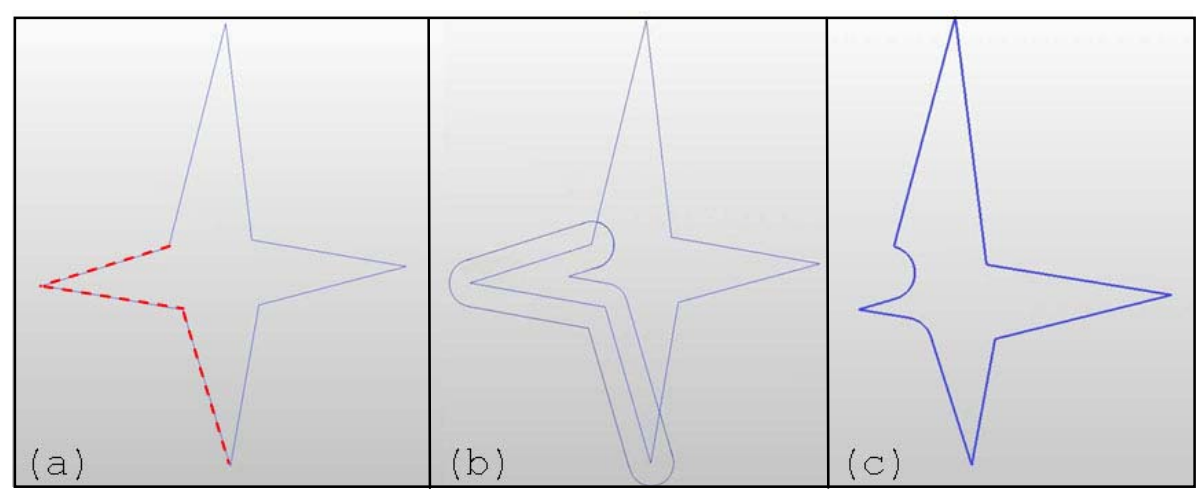

Figure 12. Example of regularized partial erosion in two dimensions. a) Trajectory to be eroded. b) Dilation of the trajectory. c) Frontier of the regularized partial erosion

In more complex objects multiple partitions may occur, which can lead to discontinuities in the final object. These discontinuities are intrinsically handled by the method, so they are not special cases in fact. Some of these cases are shown in figure 13: 


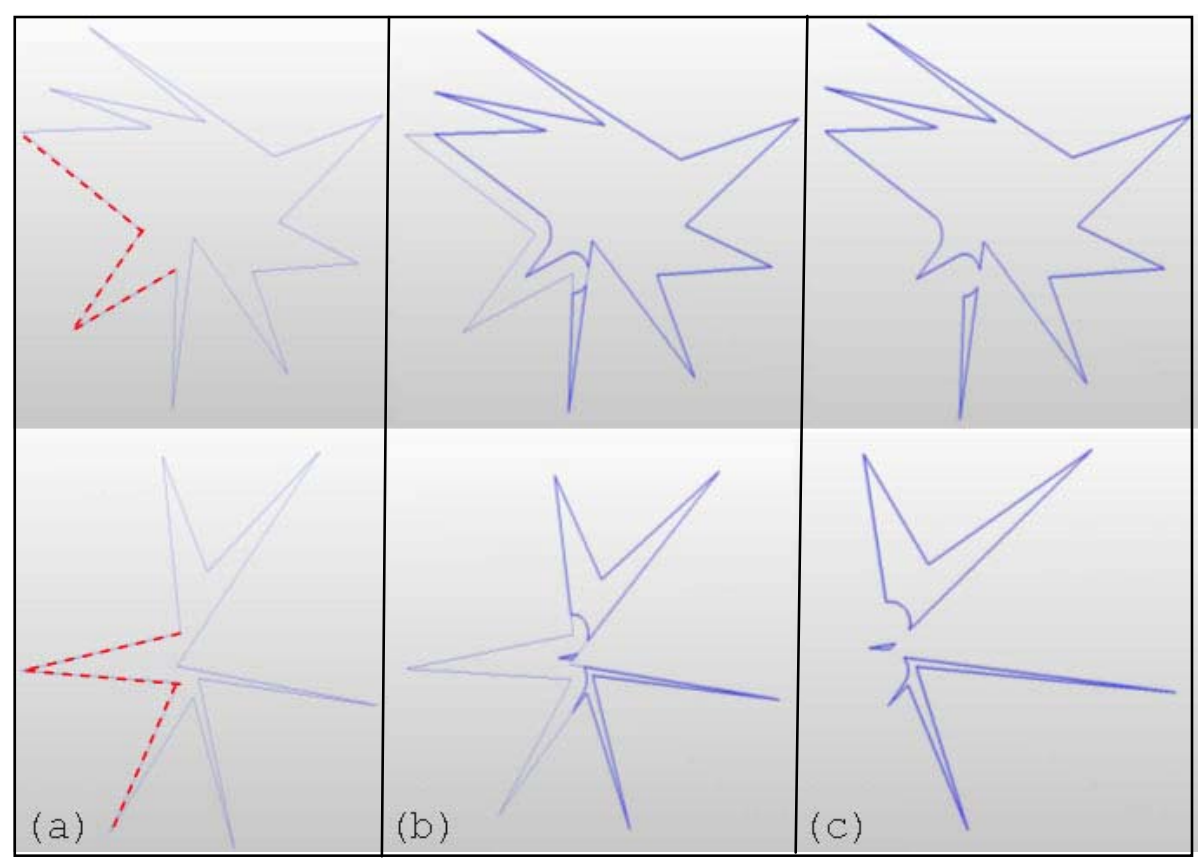

Figure 13. Examples of regularized partial erosions in two dimensions with discontinuities. a) Trajectories to be eroded. b) Dilation of the trajectory. c) Frontiers of the regularized partial erosions

\section{Secondary three-dimensional model}

For the three-dimensional erosion the same concepts of two-dimensional erosion have been applied, adapting it to the three dimensions subsequently. While in the twodimensional dilation of an erosion path only one direction of rotation is considered at the path extremes, in three dimensions two senses of rotation must be calculated, side and front, giving the dilation a three-dimensional treatment through a cylindrical shape. To represent the three-dimensional dilation, a lateral rotational sweep is done 
for each point belonging to the segment. For the extreme points a frontal rotational sweep is also done. The lateral sweep produces perpendicular arches to the segment and the frontal sweep produces hemispheres (figure 14).
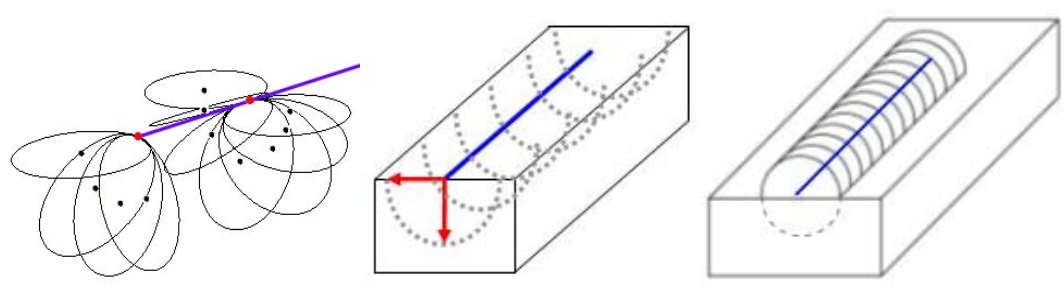

Figure 14. Dilation of a three-dimensional trajectory

As in two dimensions, in three dimensions it is not necessary to calculate the whole dilation of the trajectory, because only the points inside the element to be eroded participate in the erosion. The algorithm to obtain the geometric representation of the three-dimensional morphological erosion is shown in figure 15. 


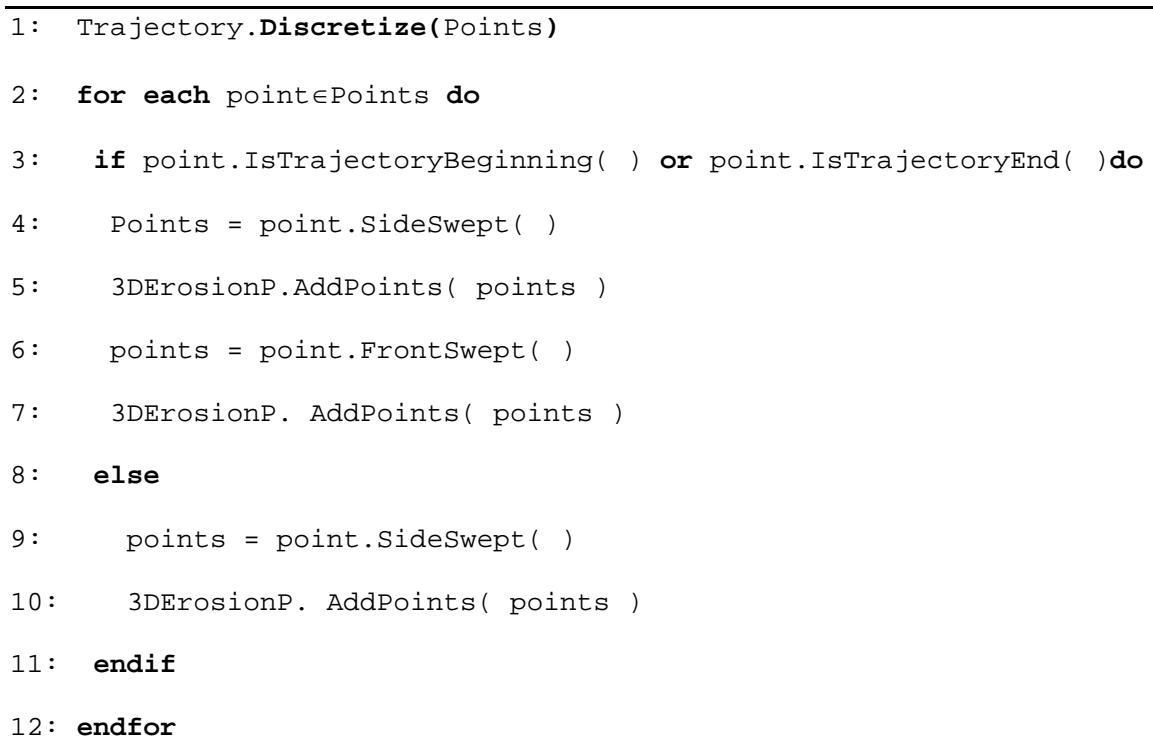

Figure 15. Partial erosion algorithm in three dimensions

At this point, the frontier of the dilation is obtained (figure 16). Nevertheless, the whole regularized erosion needs a further step: subtracting this dilation frontier from the frontier of the original object. This way, the sum of all the surfaces constitutes a single object with solid appearance. This model of representation is not made up of Boolean operations between solids, but it is a sum of surfaces: the surfaces of the initial shape that are not involved in the erosion, some portions of the surfaces of the initial shape that are involved in the erosion (obtained by a process of cut) and, finally, the surface of the dilation generated from the arches as explained before. 


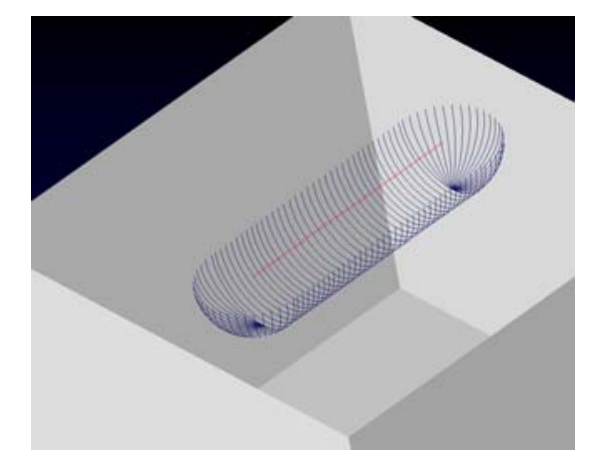

Figure 16. Dilation surface in form of arches as obtained from the algorithm

Therefore, a first step to get the whole superficial representation of the object is to obtain the cut surface from the original object surface and the erosion trajectory. For this, the erosion contour is used as the profile to cut of the original surface, so that the obtained cut surface will be part of the final eroded object (figure 17). 


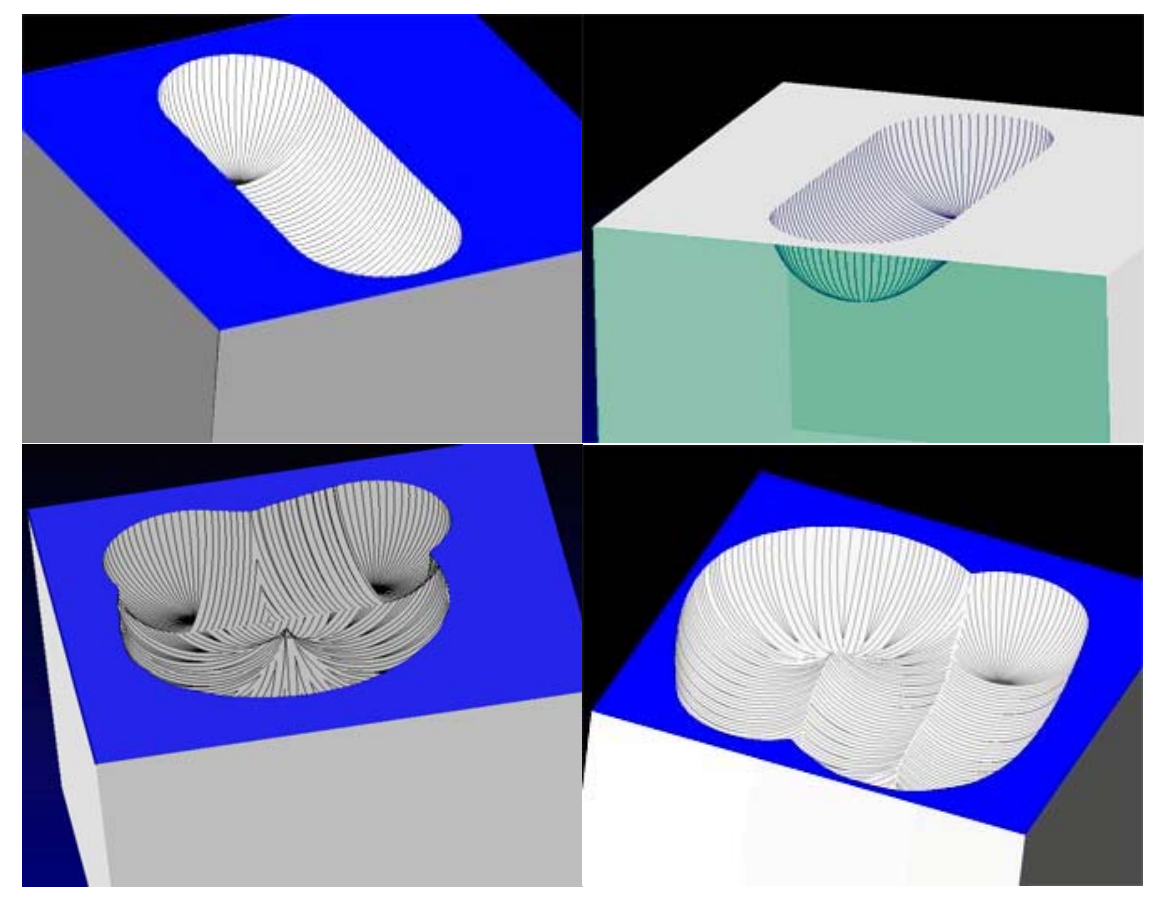

Figure 17. Cut surfaces 


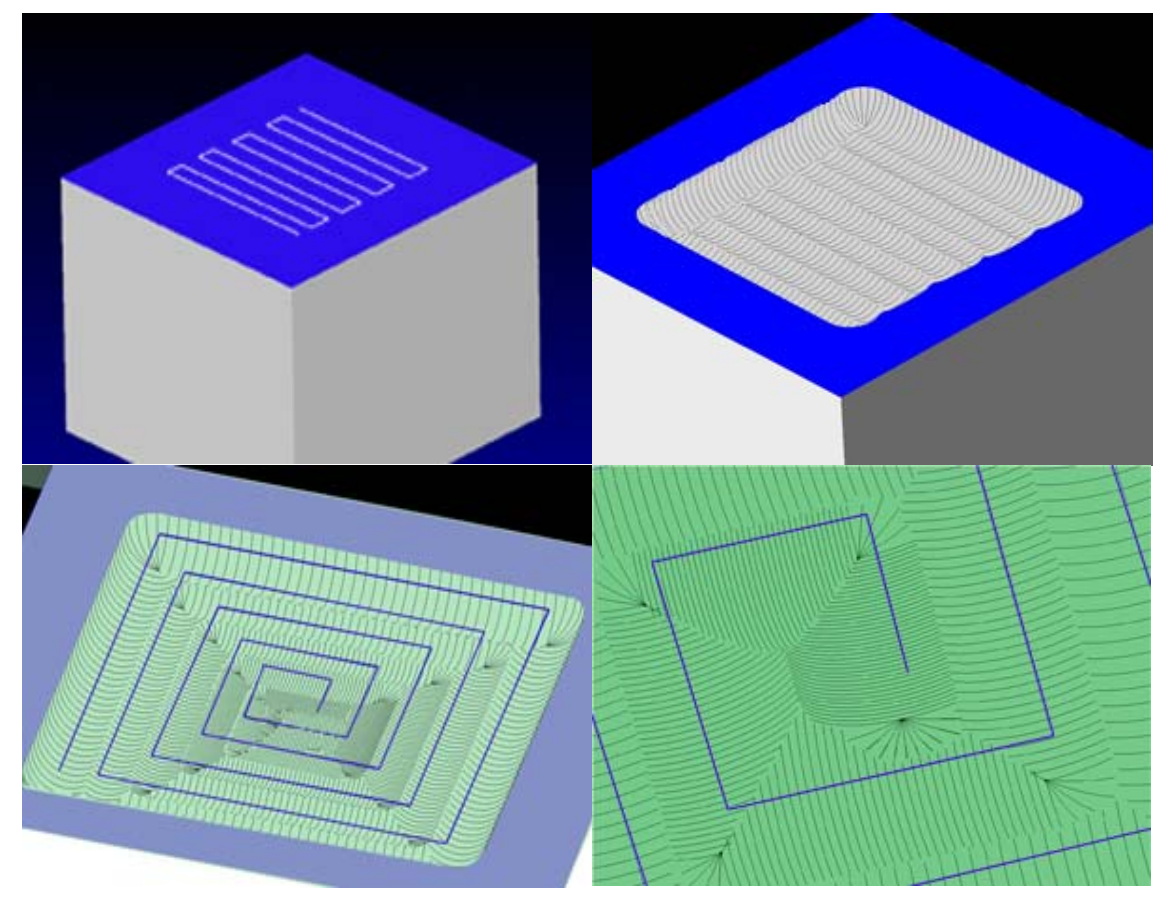

Figure 18. Details trajectories of erosion

The last step of the erosion process is the generation of the erosion surface from the arches of dilation. As the generation of the dilation arches is done in an ordered manner, the erosion surface can be obtained just triangulating point to point the consecutives arches of the trajectory. The calculation of the triangles is, therefore, immediate, just joining the consecutive arches that are obtained in order. This calculation is accurate and very fast, since most graphics cards on the market are optimized for drawing triangles. Once the erosion surfaces are generated, they are added to the previously obtained cut surfaces and the other original surfaces to obtain together the result of the regularized partial erosion of a solid object (figure 18, 19, and 20). 


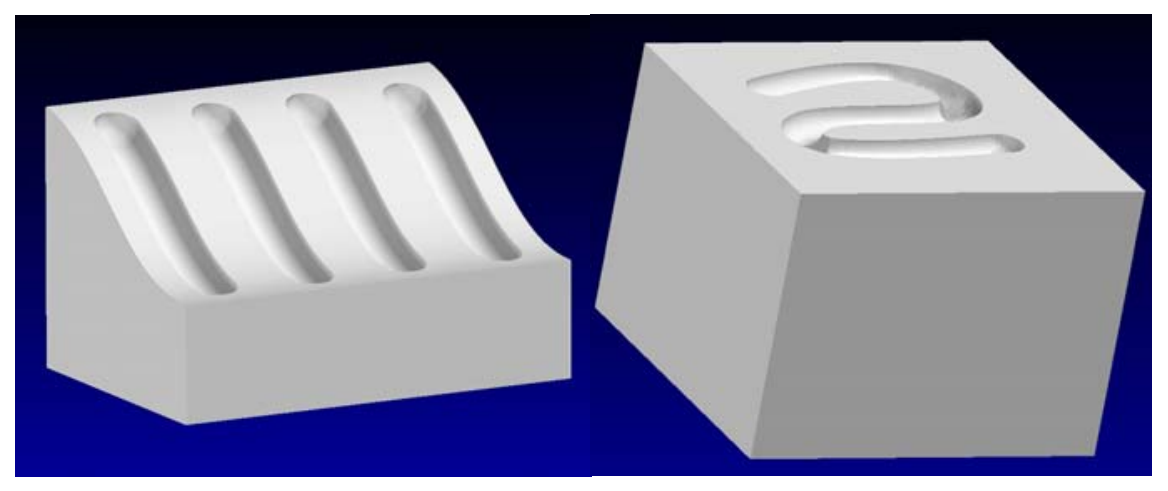

Figure 19. Examples of regularized partial erosions in three dimensions.
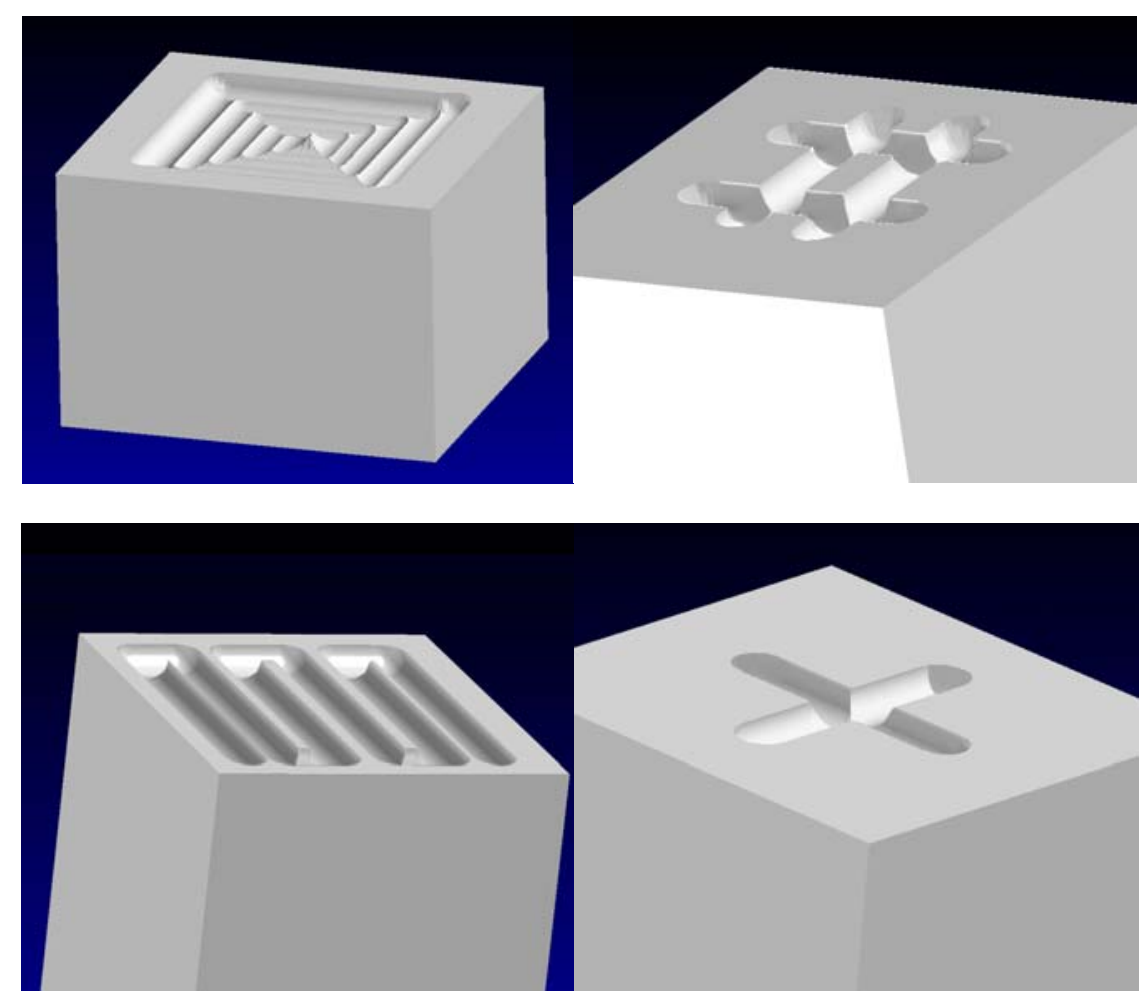

Figure 20. Examples of regularized partial erosions in three dimensions. 
The use of a superficial model is crucial, because it allows a direct representation. As a consequence, the method is very quickly and accurate, due to the lack of extra calculations for the erosion trajectories visualization. The process of display of the object machining has a low temporal cost, as can be seen in the chart in figure 21 . It shows the execution time for a simple object like the one in figure 16 . The platform is based on Microsoft Windows XP® working on an Intel Pentium IV ${ }^{\circledR}$ processor at 1.8 Mh with $2 \mathrm{~Gb}$ of RAM memory. From the graph it can be concluded that the quality of the surface that determines the temporal complexity of the model and that the accuracy of the trajectory do not cause substantial differences in execution time.

The algorithm to obtain the geometric representation of the three-dimensional morphological erosion is shown in figure 15.

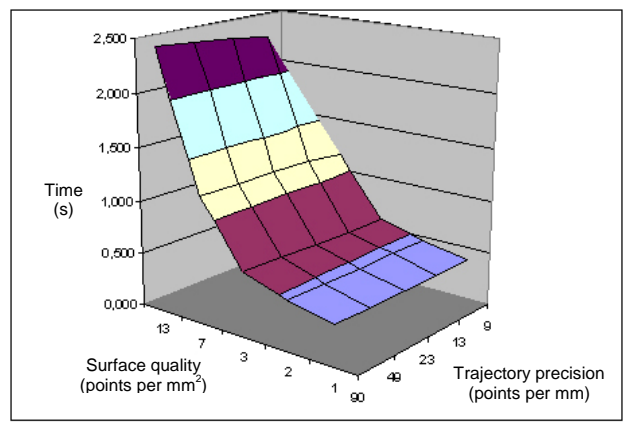

Figure 21. Execution time for a simple erosion (fig 16). 


\section{Conclusions}

The model addresses the process of designing objects by assimilation of the machining process. It gives solution to the design of complex objects and an arithmetic support to the generation of manufacturing trajectories, one of the most complex problems that are currently treated in the CAD / CAM. In fact, it is possible to establish an analogy between the design process and the craft work of a sculptor designing their pieces by hand with its tools. As a specific model for the machining process, it gives a direct solution to the problem of trajectories generation since they are already defined at the design phase.

The proposed model is based on set theory and mathematical morphology and no operation between solids or surfaces is done. All the objects in this model are just point sets, and so, the operations are done exclusively between point sets. This way complex geometric operations such as surface intersection or Boolean operations, which are characteristic of other models such as B-Rep or CSG, are avoided. As a result, the model is generic and robust, as it will be explained in the following paragraphs.

The proposed model is generic, as it can be extended to any other tool shape, because the structuring element (or tool) is a point subset, so there are no constraints in the shape it can adopt. In fact, in [7] the model was extended to other tool shapes such as squared-end tools, corner radius tools, toroidal tools as used in shoe last manufacturing [14], and conical tools used for pencil tracing for three dimensions.

The model is also robust in two aspects. On one hand, the internal representation is based on set theory, that is, every piece is stored as a set of points. The operations 
between point sets are very simple and no complex geometric calculation is needed. In fact, every high level operation (such as the erosion or dilation operation) is based on a sequence of an instant basic operation. The only geometric calculations implied in the basic operations are a transformation and the Euclidean distance. On the other side, the surface calculation needed to display the objects, is done by a simple triangulation method that homogeneously deals with every point in the set representing the piece. This method avoids special cases or incorrect solutions such as numerical errors, degenerated triangles and so on.

The formal morphological model allows the generation of any complex object that can be mechanized. Nevertheless, to make the model practical it is necessary to obtain the boundaries of the designed object, that is, the surface model for representation. One of the most important values of this model is, indeed, the way the representation is done. The actual calculation of the erosion surface results in the direct visualization of the object, that is, there are no intermediate steps between the erosion calculation and its display. This way, the model succeeds in a very quick and faithful representation of the eroded object. The secondary model allows the user to check the validity of the object modeling, since it exactly represents the result of machining.

To implement the model of representation the problem in two dimensions has been addressed firstly, and then the same concepts have been applied to the representation of three-dimensional objects.

The limited resources required to store the basic geometric structures and to operate between point sets make the model light to be computed, so that no powerful computers for solving complex problems of erosion are needed. 
The generation of the erosion surface by the triangulation of the trajectories points greatly facilitates the optimization of the visualization and the use of textures. It also offers important functions for the actual representation of the scene such as wire representation, face occlusion and so on, aspects that will give a new dimension to actual display of the scene. Besides, as the user has control on the definition of the surfaces, it is possible to identify some areas where, because of the peculiarities of the object, it is necessary to have greater precision.

In a future research, it is planned to deal with the use of more complex structuring elements (i.e. other tool shapes in three dimensions) and the application of the proposed model on actual CAD / CAM systems.

\section{References}

[1] Choi, B. K., 1991, Surface Modeling for CAD/CAM. Elsevier Science Publishers, Amsterdam.

[2] Iglesias, A., Gálvez, A., Puig-Pey, J., 2001, “Computational Methods for Geometric Processing. Applications to Industry”, Lecture Notes in Computer Science 2073, pp. 698707.

[3] Maekawa, T., 1999, “An overview of offset curves and surfaces”, Computer-Aided Design 31(3), pp. 165-173

[4] Serra, J., 1982, Image Analysis and Mathematical Morphology, Academic Press, London.

[5] Jimeno, A. M., Macía F., García-Chamizo J. 2004, "Trajectory-based morphological operators: a morphological model for tool path computation", Proceedings of the international conference on algorithmic mathematics \& computer science, AMCS 2004, Las Vegas (USA). 
[6] Molina-Carmona, R., Jimeno, A., Rizo, R., 2007, "Morphological offset computing for contour pocketing”, Journal of Manufacturing Science and Engineering Transactions of the ASME, 129(2), pp. 400-406.

[7] Molina-Carmona, R., Jimeno, A., Davia, M., 2008, "Contour pocketing computation using mathematical morphology”, Int J. Adv. Manuf. Technol., 36(3-4), pp. 334-342.

[8] Molina-Carmona, R., 2002, Modelo Superficie - Trayectoria. Un modelo geométrico para el diseño y la fabricación de objetos tridimensionales, $\mathrm{PhD}$ Thesis, Universidad de Alicante, Alicante (Spain).

[9] Kim, S.J., Yang, M.Y, 2005, ”Triangular mesh offset for generalized cutter”, ComputerAided Design, 37(10), pp. 999-1014.

[10] Meyer, F., 2001, “An overview of morphological segmentation”, International Journal of Pattern Recognition and Artificial Intelligence, 15(7), pp. 1089-1118.

[11] Pujol, F.A., García Chamizo, J.M., Fuster, A., Pujol, M., Rizo, R., 2002, "Use of Mathematical Morphology in Real-Time Path Planning", The International Journal of Systems \& Cybernetics, 31(1), pp. 115-123.

[12] Vincent, L., 1993, Morphological algorithms, E. R. Dougherty editor, Mathematical Morphology in Image Processing, Marcel Dekker.

[13] Jimeno, A. M., 2003, Modelado topológico del proceso de fabricación. Aplicación al compensado de herramienta, $\mathrm{PhD}$ Thesis, Universidad de Alicante, Alicante (Spain).

[14] Jimeno A, García J., Salas F. 2001. Shoe lasts machining using Virtual Digitizing. International Journal of Advanced Manufacturing Technology. 17(10), pp.744-750. 